\title{
ROBUST CONSTANT MODULUS ARRAYS BASED ON FRACTIONAL LOWER-ORDER STATISTICS
}

\author{
Marilli Rupi, Panagiotis Tsakalides, Chrysostomos L. Nikias; and Enrico Del Re ${ }^{\dagger}$ \\ Signal \& Image Processing Institute \\ Department of Electrical Engineering - Systems \\ University of Southern California \\ Los Angeles, CA 90089-2564 \\ e-mail: tsakalid@sipi.usc.edu
}

\begin{abstract}
This paper addresses the problem of blind equalization for digital communications using an array of sensors at the receiver to copy constant modulus signals in the presence of heavy-tailed additive channel noise. First, we demonstrate the negative effects of channel noise to the original CMA cost function in terms of convergence. Then, we introduce a new CMA criterion based on the fractional lower-order statistics (FLOS) of the received data. The proposed criterion is able to mitigate impulsive noise at the receiver and at the same time restores the constant modulus character of the transmitted communication signal. We perform an analytical study of the properties of the new cost function and we illustrate its convergence behavior through computer simulations.
\end{abstract}

\section{INTRODUCTION}

In digital communication systems, the antenna receiver must be able to estimate the information sequence in severe interference backgrounds. As a result, the problem of cochannel signal suppression has been the focus of considerable research in the signal processing and communications communities. In the mid sixties, Lucky and Austin developed a technique for sub-optimal receivers which used linear equalizers in order to bring the filter output as close as possible to the transmitted symbol sequence $[1,2]$. In addition, Widrow introduced an adaptive equalizer which automatically adjusts the filter coefficients to optimize a specified performance index and to compensate for time variations in the channel characteristics [3]. The blind equalizer proposed by Sato in the seventies used the same main concept of updating the weights, but it was based on an initial adjustment of the coefficients without the benefit of a training sequence [4].

The Constant Modulus Algorithm (CMA) was studied by Treichler and Larimore [5, 6] who analyzed its performance in terms of capture and lock behavior. Initial CMA

"The work of the second and third authors was supported by the Office of Naval Research under Contract N00014-92-J-1034

'Laboratorio Elaborazione dei Segnali e Telematica, Dipartimento di Ingegneria Elettronica, Universitá degli Studi di Firenze, Firenze, FI 50139, ITALY studies considered only the temporal diversity at the receiver. The development of advanced division access techniques has made the concept of spatial diversity worthpursuing. As a consequence, directional array antenna beamformers have taken the place of omnidirectional antennas. In this context, Gooch and Lundel introduced the so-called constant modulus array, which exploits the constant modulus properties of the communication signal of interest to steer a beam in the direction of the information sequence while placing nulls in the directions of interferences [7].

Most of the theoretical work on blind equalizers based on the CM criterion has assumed the absence of additive channel noise or it has focused on the case where the channel noise is assumed to follow the Gaussian model [8]. The Gaussian assumption is frequently motivated because it often leads to mathematically tractable solutions. However algorithms designed under the Gaussian assumption exhibit various degrees of performance degradation, depending on the non-Gaussian nature of the environment. For this reason, there is a need to use more general and realistic non-Gaussian models and design robust equalization techniques that take into account the possible heavy-tail nature of the data. Indeed, experimental results have been reported where electromagnetic noise in urban mobile-radio channels is heavy-tailed in nature and cannot be modeled by means of Gaussian or other exponential-tailed distributions $[9,10]$. In addition, impulsive channels appear in telephone lines, underwater acoustic communications (icecracks), atmospheric environments (thunderstorms), and mobile communications.

The presence of heavy-tailed noise together with the desired signal has a negative effect on the conventional CMA performance in terms of its convergence behavior. Our work is devoted to the development of a novel constant modulus array signal processing algorithm for robust performance in the presence of interference/noise environments that can be modeled according to the alpha-stable law. The new method is based on the constant modulus property of the Fractional Lower-Order Statistics (FLOS) of the signal of interest and is able to handle robustly the presence of heavytailed noise and interference in the data. In the absence of noise, the performance of the proposed method is the same with that of the original CMA. 


\section{CONSTANT MODULUS ARRAYS BASED ON FLOS}

\subsection{Statistical Models for Heavy-Tailed Noise}

Man-made as well as natural physical processes can generate interferences containing noise components that are impulsive in nature. In modeling this type of signals the symmetric alpha-stable $(S \alpha S)$ distribution provides an attractive theoretical tool. It was proven that under broad conditions, a general class of heavy-tailed noise follows the stable law [11].

The $S \alpha S$ class of distributions is best defined by its characteristic function:

$$
\varphi(\omega)=\exp \left(\jmath \delta \omega-\gamma|\omega|^{\alpha}\right),
$$

where $\alpha$ is the characteristic exponent restricted to the values $0<\alpha \leq 2, \delta(-\infty<\delta<\infty)$ is the location parameter, and $\gamma(\gamma>0)$ is the dispersion of the distribution. The dispersion parameter $\gamma$ determines the spread of the distribution around its location parameter $\delta$, much in the same way that the variance of the Gaussian distribution determines the spread around the mean. The characteristic exponent $\alpha$ is the most important parameter of the $S \alpha S$ distribution and it determines the shape of the distribution. The smaller the characteristic exponent $\alpha$ is, the heavier the tails of the alpha-stable density. It is this heavy-tail characteristic that makes the alpha-stable densities appropriate for modeling noise that may be impulsive in nature. We should also note that the stable distribution corresponding to $\alpha=2$ coincides with the Gaussian density.

The appeal of $S \alpha S$ distributions as statistical models derives from some important properties. They: (i) naturally arise as limiting processes via the Generalized Central Limit Theorem; (ii) possess the stability property and share many features with the Gaussian density such as unimodality, symmetry with respect to the location parameter, bellshape; (iii) possess finite moments of order $p$ only when $p$ is strictly less than $\alpha$ : $E|X|^{p}<\infty$ for $p<\alpha$.

\subsection{Blind Equalization with CMA Arrays}

The constant modulus family of blind equalizers is based on a cost function that assigns a penalty to deviations in the modulus of the controller's complex output signal. The cost function is given by

$$
J_{p q}^{C M}=E\left[\left|\|y(n)\|^{p}-\delta\right|^{q}\right]
$$

where $E[\cdot]$ denotes statistical expectation, $y(n)$ is the controller output at time $n, p$ and $q$ are positive integers, and $\delta$ is a constant greater than zero.

The most famous member of this family is the Constant Modulus Algorithm (CMA) for which both parameters $p$ and $q$ are equal to two $[5,6]$. CMA has been used in a fractionally spaced context. Recent work has exploited channel diversity to achieve perfect equalization in noise free environments. Initially, channel diversity referred mainly to the temporal domain and was produced by oversampling the received analog signal in time. But spatial diversity has also been used by employing an array of sensors at the receiver [7].
Consider an array of $N$ equispaced sensors, which receive signals generated by $Q$ sources located at $\vartheta_{1}, \ldots, \vartheta_{Q}$. Assuming the signal bandwidth to be narrow as compared to the inverse of the travel time across the array, it follows that, by using a complex envelop representation, the array output can be expressed as:

$$
\mathbf{x}(t)=\mathbf{A}(\vartheta) \mathbf{s}(t)+\mathbf{n}(t),
$$

where $\mathbf{x}(t)=\left[x_{1}(t), \ldots, x_{N}(t)\right]^{T}$ is the array output vector; $\mathrm{s}(t)=\left[s_{1}(t), \ldots, s_{Q}(t)\right]^{T}$ is the signal vector received by the reference sensor of the array; $\mathbf{A}(\vartheta)$ is the $N \times Q$ steering matrix, whose $r$ th column vector $\mathbf{a}\left(\vartheta_{r}\right)$ is $\left[1, e^{-j 2 \pi(d / \lambda) \sin \vartheta_{r}}, \ldots\right.$ $\left.e^{-\jmath(N-1) 2 \pi(d / \lambda) \sin \vartheta_{r}}\right]^{T}$ and $\mathbf{n}(t)=\left[n_{1}(t), \ldots, n_{N}(t)\right]^{T}$ is the noise vector.

The CMA attempts to minimize the cost function shown in (2) by following the path of steepest descent. By letting the array weights at time $n$ be given by the vector $w(n)$, the update equation for the $p=2$ and $q=2$ case is given by

$$
\begin{aligned}
\mathrm{w}(n+1) & =\mathrm{w}(n)-\mu \mathrm{x}(n) \epsilon^{*}(n) \\
\epsilon(n) & =y(n)\|y(n)\|^{2}-y(n) \\
y(n) & =\mathbf{w}^{H}(n) \times(n)
\end{aligned}
$$

\subsection{The FLOS-CMA Array}

The main characteristic associated with the classical CMA is that it involves fourth-order moments of the signal. In the presence of heavy-tailed noise, the use of second- or higherorder statistics in effect amplifies the noise. For such cases, we propose a new cost function that considers information of constant modulus regarding the communication signals and uses FLOS to mitigate the impulsive noise component. The new cost function has the expression

$$
J_{p, q}^{F L O S-C M A}=E\left[\left|\|y(n)\|^{(p-1)} y(n)-\delta y(n)\right|^{q}\right]
$$

where $y(n)$ is the array output and $\delta$ is a real representing the constant signal modulus. The pair $(p, q)$ takes values, possibly fractional, between 0 and $\alpha$, where $\alpha$ is the characteristic exponent of the alpha-stable distribution that best describes the statistics of the noise vector $n(t)$. Furthermore, the product $p \cdot q$ must be less than $\alpha$.

First, we develop the recursive update formula for the array weights using the principle of steepest descent to minimize the proposed cost function in (7) (for a complete treatment see [12]).

$$
\begin{aligned}
& \mathbf{w}(n+1)=\mathrm{w}(n)-\mu \nabla_{w \cdot J_{p q}} \\
& \quad=\mathbf{w}(n)-\mu \frac{q}{2}\|\vec{A}\|^{q-2} \mathbf{x}(n)\left(p\|y(n)\|^{p-1}-1\right) \bar{A}^{*}(8)
\end{aligned}
$$

where

$$
\bar{A}=y(n)\left(\|y(n)\|^{p-1}-1\right)
$$

Naturally, the convergence behavior of the FLOS-CMA algorithm is an important issue when more that one constant modulus signals are present. In [12], we address the lock and capture properties of the new criterion by finding the expression of the curve boundaries that divide the two zones in positive lock zone and positive capture zone. Figure 1 


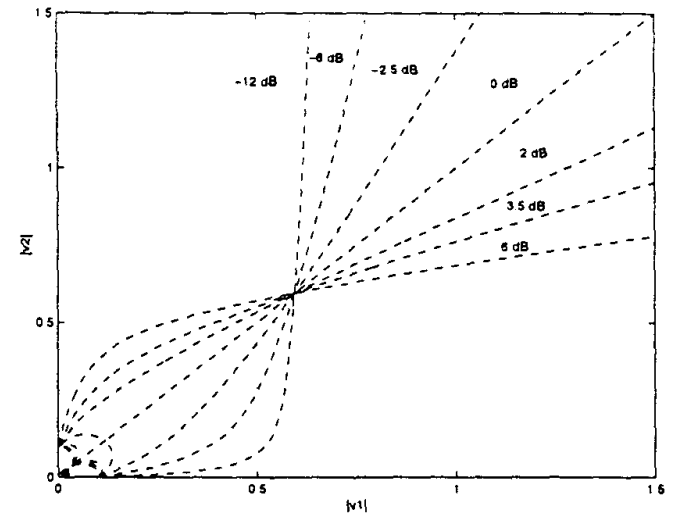

Figure 1: FLOS-CMA: Lock and capture boundaries for different SIR values.

depicts the lock and capture boundaries for a $\mathrm{CM}$ signal and a $C M$ interference, for several signal-to-interference ratios (SIR). The FLOS-CMA array locks onto the CM signal of strongest initial power at the array output.

\subsection{Experimental Results}

In this section, we test and validate the new FLOS-CMA adaptive beamformer and compare its performance with that of the conventional CMA array in a noisy environment. Four transmitted signals impinge on the array from directions $\theta=\left[30^{\circ},-40^{\circ}, 60^{\circ},-15^{\circ}\right]$. The number of snapshots available to the array is $M=10,000$. The SNR is $10 \mathrm{~dB}$ and the noise component is modeled as an alphastable process with $\alpha=1.85$, i.e., the noise is fairly close to Gaussian. We plot the output path of each signal (cf. Figure 2), the beampatterns after convergence (cf. Figure 3), and the constellation plot of the array output signal at convergence (cf. Figure 4) for both the original CMA and the proposed FLOS-CMA algorithms.

Figure 2 demonstrates that occurrences of noise outliers during the adaptation, have an adverse affect to the learning curve of the original CMA method. On the other hand, the proposed FLOS-CMA cost function can suppress the noise components and results in a much smoother learning curve. Naturally, the improved performance of the FLOS-CMA is also demonstrated by comparing the adapted beampatterns in Figure 3. It is apparent that only the FLOS-CMA array places nulls in the directions of the three multipath signals at $-40^{\circ}, 60^{\circ}$, and $-15^{\circ}$. Finally, Figure 4 demonstrates that the received vectors of the adapted FLOS-CMA method are tightly clustered about the four constellation points, while the signal constellation of the original CMA appears as a random scatter of points.

\section{DISCUSSION}

We proposed a new method for blind equalization of communication signals using a constant modulus criterion based on fractional lower-order statistics. The introduced FLOSCMA array exploits the constant modulus property of the signal of interest and uses the heavy-tailed noise suppression capabilities of FLOS to steer a beam in the direction

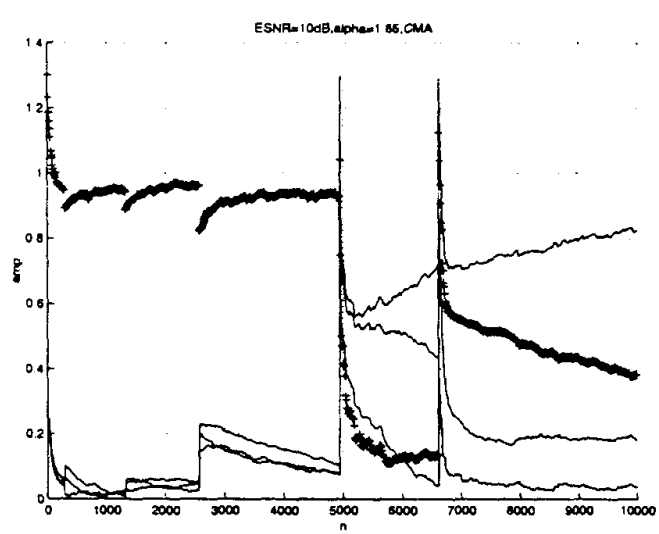

(a)

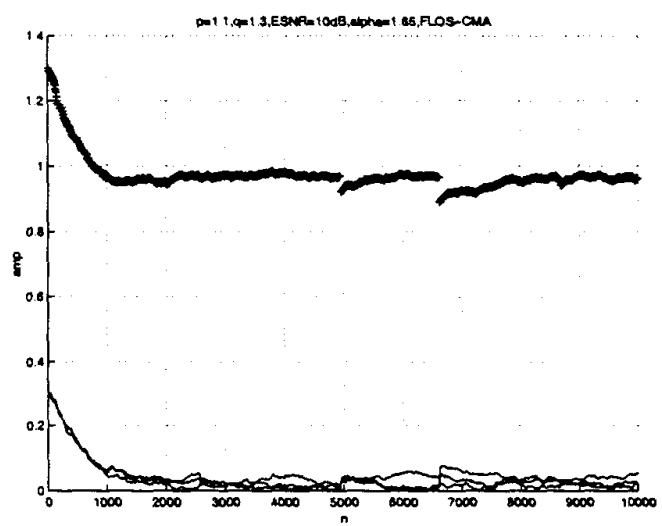

(b)

Figure 2: Convergence behavior of output amplitudes. (a): CMA, (b): FLOS-CMA.

of the signal while suppressing interference and noise. The main advantage of the proposed method is its robustness in the presence of various noise environments. Truly, by changing the parameters $p$ and $q$ in the criterion in (7) we obtain a class of FLOS-based CMA beamformers which provides considerable flexibility that can be useful for optimization purposes in the presence of nonstationary noise environments.

The proposed method developed using FLOS has approximately the same computational complexity as the existing CMA methods. The additional computational load is due to the need for calculating a fractional power $(p<2)$ rather than a square power. The technique can be used in commercial communication applications in which impulsive channels tend to produce large-amplitude interferences and sharp noise spikes more frequently than what is expected from Gaussian channels. 


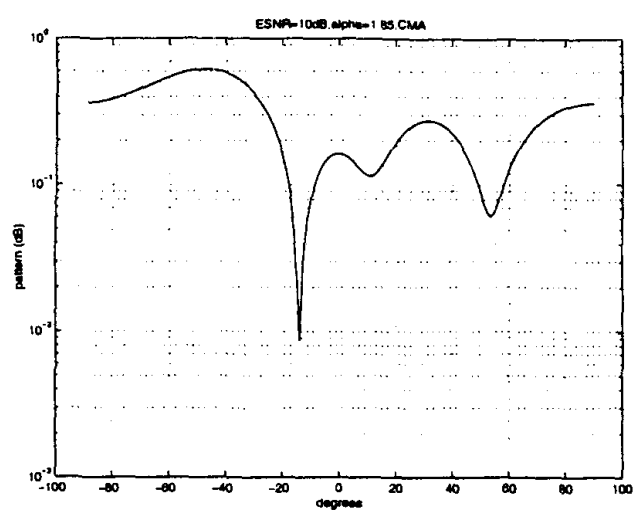

(a)

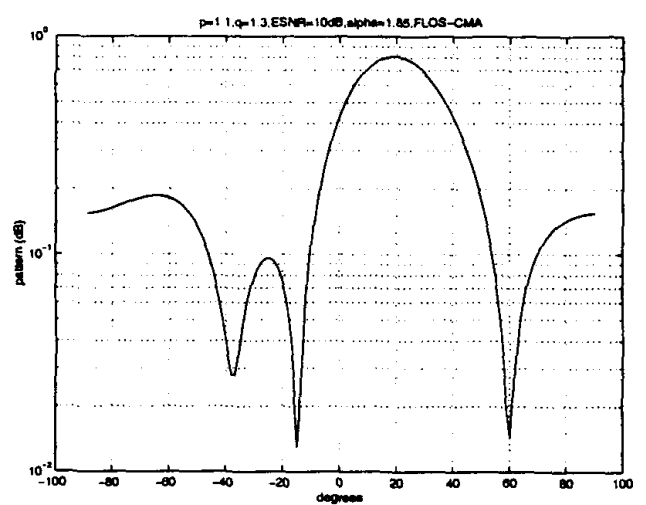

(b)

Figure 3: Beampattern of the array after adaptation. (a): CMA, (b): FLOS-CMA.

\section{REFERENCES}

[1] R. W. Lucky, "Automatic equalization for digital communications," tech. rep., Bell Syst. Tech. J., 1965.

[2] M. E. Austin, "Decision-feedback equalization for digital communication over dispersive channel," tech. rep., MIT Lincoln Laboratory, Lexington, Mass., 1967.

[3] B. Widrow, "Adaptive filters, 1: Fundamentals," tech. rep., Stanford Electronics Laboratory, Stanford University, Stanford, Calif., 1966.

[4] Y. Sato, "A method of self-recovering equalization for multilevel-amplitude modulation systems," IEEE Trans. on Comm., June 1975.

[5] J. Treichler and B. Agee, "A new approach to multipath correction of constant modulus signals.," IEEE Trans. on ASSP, Apr. 1983.

[6] J. Treichler and M. Larimore, "New processing techniques based on the constant modulus adaptive alga rithm," IEEE Trans. on ASSP, Apr. 1985.

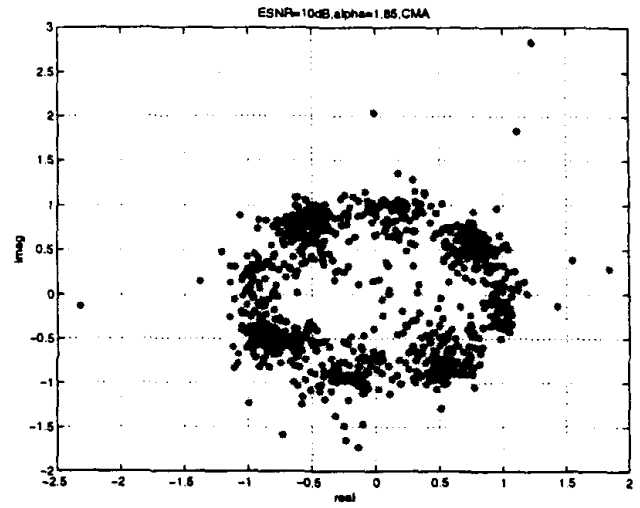

(a)

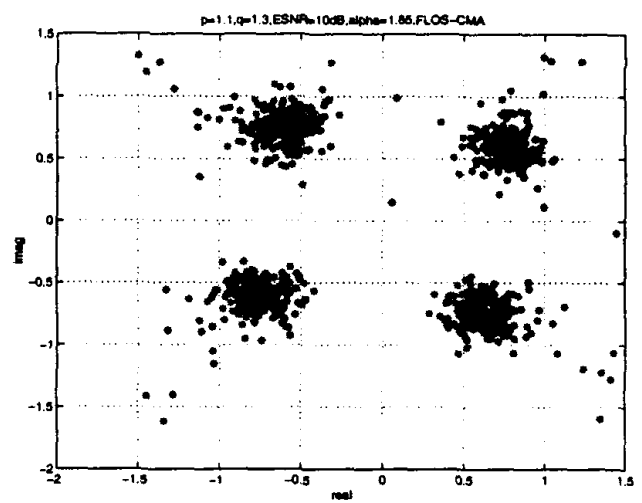

(b)

Figure 4: Signal constellation pattern of array output after convergence. (a): CMA, (b): FLOS-CMA.

[7] R. Gooch and J. Lundell, "The cm array: an adaptive beamformer for constant modulus signals.," in Proc. ICASSP, 1986.

[8] I. Fijalkow, A. Touzni, and J. Treichler, "Fractionally spaced equalization using cma: Robustness to channel noise and lack of disparity.," IEEE Trans. on Signal Proc., vol. 45, Jan. 1997.

[9] D. Middleton, "Statistical-physical models of urban radio noise environment - part i: Foundations," IEEE Trans. Elect. Comput., vol 14, pp 38-56, 1972.

[10] D. Mitra and H. Poor, "Detection of spread-spectrum signals in multi-user environment," in Proc. ICASSP, pp 1844-1847, 1995.

[11] C. L. Nikias and M. Shao, Signal Processing with Alpha-Stable Distributions and Applications. New York: John Wiley and Sons, 1995.

[12] M. Rupi, P. Tsakalides, E. D. Re, and C. L. Nikias, "A constant modulus algorithm based on fractional lowerorder statistics (FLOWERS-CMA)," IEEE Trans, on Signal Proc., Sept. 1998. submitted as a regular paper. 